\title{
Hydrogen Bonding and Mesomorphic Properties for Side Chain Type Liquid Crystalline Polyurethanes Containing Rigid Moieties in the Polymer Backbone
}

\author{
Takashi Mihara and Naoyuki KoIDE \\ Department of Chemistry, Faculty of Science, Science University of Tokyo, \\ 1-3 Kagurazaka, Shinjuku-ku, Tokyo 162, Japan \\ (Received July 24, 1996)
}

\begin{abstract}
Side chain type liquid crystalline polyurethane (SLCPU)s containing various kinds of mesogenic groups were prepared by polyaddition of a diol-monomer with $p$-phenylenediisocyanate. Three SLCPUs (PU-a, b, and c) exhibited typical mesomorphic properties. Further, the relationship between mesomorphic properties and hydrogen bonding between urethane bonds in the polymer backbone (H-bonding) for the three SLCPUs was investigated by thermally controlled FT-IR. A small decrease in the strength of $\mathrm{H}$-bonding was observed with increasing temperature. The magnitude of the decrease in the strength of H-bonding was dependent on the species of the mesogenic group.
\end{abstract}

KEY WORDS Side Chain Type Liquid Crystalline Polymer/Polyurethane/Hydrogen Bonding/FT-IR/

The mesomorphic properties of side chain type liquid crystalline polymer (SLCP)s are greatly influenced by mesogenic groups, flexible spacer length introduced between mesogenic group and polymer backbone, etc. Polyacrylic acid, polymethacrylic acid, and polysiloxane are very often employed for the polymer backbone of SLCPUs. ${ }^{1-3}$ However, the mesomorphic properties of SLCPs with a polymer backbone having the specific interaction which could be formed between the amide group in the polymer backbone like polyurethane and/ or polyamide derivatives, are not often reported. ${ }^{4,5}$ We are interested in the polymer backbone containing the specific interaction. We have already found that mesomorphic properties are exhibited for polyurethanes with a mesogenic group in the side chain regardless of rigidity or flexibility of the polymer backbone, as shown in Figure $1 .^{6}$ Furthermore, the relationship between the hydrogen bonding formed by $\mathrm{NH}$ group and $\mathrm{C}=\mathrm{O}$ group of the urethane bonds in the polymer backbones (H-bonding) and mesomorphic properties for the SLCPUs was investigated by thermally controlled FT-IR measurements. ${ }^{6-8}$ In the case of the SLCPUs containing a flexible alkyl chain in the polymer backbone (flexible SLCPU), a nematic phase was exhibited with a remarkable decrease in the strength of $\mathrm{H}$-bonding, while the thermal dependence in the strength of $\mathrm{H}$-bonding for SLCPUs with a rigid moiety in the polymer backbone (rigid SLCPU) was not observed. Thus the relationship between mesomorphic properties and $\mathrm{H}$-bonding for the flexible SLCPU was clarified, however, that for the rigid SLCPU was not clarified by the FT-IR measurements.

In this paper, in order to investigate the effect of the $\mathrm{H}$-bonding on the mesomorphic properties, SLCPUs with various kinds of mesogenic group were synthesized. The thermal properties for the SLCPUs were examined by polarizing optical microscopy, differential scanning calorimetry (DSC), and wide angle X-ray scattering (WAXS) measurements. Furthermore, the relationship between mesomorphic properties and $\mathrm{H}$-bonding for the SLCPUs was also investigated by thermally controlled FT-IR measurements.

\section{EXPERIMENTAL}

\section{Materials}

The rigid SLCPUs with various kinds of mesogenic groups were synthesized from diol-monomers and $p$ phenylenediisocyanate. Diol-monomers were synthesized according to Scheme 1. One example of the method of diol-monomer synthesis is shown.

p-(6-Bromohexyloxyphenylazo)nitrobenzene (2-a). 1,6dibromohexane $\left(9.0 \mathrm{~g}, 3.7 \times 10^{-2} \mathrm{~mol}\right)$, potassium carbonate $\left(2.0 \mathrm{~g}, 1.5 \times 10^{-2} \mathrm{~mol}\right)$ and a small amount of potassium iodide were added to a solution of acetone containing $p$-(4-hydroxyphenylazo)nitrobenzene $(3.0 \mathrm{~g}$, $\left.1.2 \times 10^{-2} \mathrm{~mol}\right)$. The mixture was refluxed for 9 hours. After the precipitation was removed by filtration, the filtrate was evaporated to dryness under reduced pressure. After a large excess of cold hexane was added to the mixture to remove a excess of 1,6-dibromohexane, the residue was filtered off. The product was obtained in a $56 \%$ yield.

${ }^{1} \mathrm{H}$ NMR $\left(\mathrm{CDCl}_{3}\right): \delta 1.3-2.0\left(\mathrm{~m} ; 8 \mathrm{H},-\left(\mathrm{CH}_{2}\right)_{4}-\right), 3.5$ $\left(\mathrm{t} ; 2 \mathrm{H}, \mathrm{BrCH}_{2}\right), 4.1\left(\mathrm{t} ; 2 \mathrm{H}, \mathrm{OCH}_{2}\right), 7.0-8.5(\mathrm{~m} ; 8 \mathrm{H}$, Ar-H)

IR (KBr): $v$ 1600, $1500(\mathrm{Ar}), 1585(\mathrm{~N}=\mathrm{N}), 3000$ $2850(\mathrm{CH})$

$2,2^{\prime}-[N-[6-[(4-N i t r o p h e n y l a z o]$ phenyloxy $]$ hexyl $]-$ iminodiethanol (3; DM-a). 2-propanol solution containing 2,2'-iminodiethanol and a small amount of potassium carbonate were added to solution containing 2-a (1.0 g: $\left.2.5 \times 10^{-3} \mathrm{~mol}\right)$. The mixture was refluxed for 14 hours.

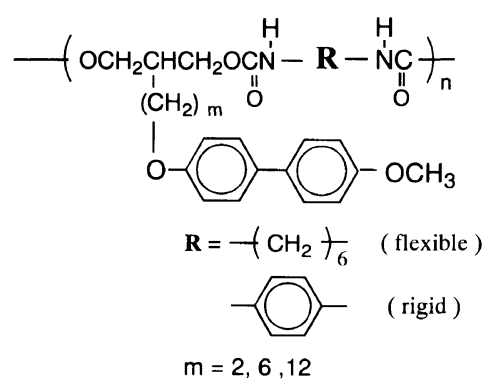

Figure 1. Structure of side chain type liquid crystalline polyurethanes. 
After the precipitation was removed by filtration, the filtrate was concentrated. The residue was poured into water and the obtained orange solid was washed with water until neutral water was obtained. The product was obtained in a $94 \%$ yield.

${ }^{1} \mathrm{H}-\mathrm{NMR}\left(\mathrm{CDCl}_{3}\right): \delta \quad 1.3-2.0\left(\mathrm{~m} ; 8 \mathrm{H},-\left(\mathrm{CH}_{2}\right)_{4}-\right)$, $2.3-2.7\left(\mathrm{~m} ; 6 \mathrm{H}, \mathrm{N}\left(\mathrm{CH}_{2}\right)_{3}\right), 2.8(\mathrm{~s} ; 2 \mathrm{H}, \mathrm{OH}), 3.7(\mathrm{t}$; $\left.4 \mathrm{H}, \mathrm{CH}_{2} \mathrm{OH}\right), 4.1\left(\mathrm{t} ; 2 \mathrm{H}, \mathrm{OCH}_{2}\right), 7.0,8.0-8.4(\mathrm{~m} ; 8 \mathrm{H}$, Ar-H)

IR (Nujol): $v 3419$ (OH), 1603, 1500 (Ar), 1579 $(\mathrm{N}=\mathrm{N})$

Polyurethanes (Polymerization). 2,2'-iminodiethanol derivatives (diol-monomer $0.5 \mathrm{~g}$ ) and 1.2 times the amount of $p$-phenylenediisocyanate were placed in the reaction vessel under a nitrogen atmosphere. After $3 \mathrm{ml}$ of distilled DMF was added to the reaction vessel, the reaction mixture was heated to $90^{\circ} \mathrm{C}$ and stirred for 24 hours under a nitrogen atmosphere. The solution was poured into $250 \mathrm{ml}$ of methanol to precipitate the polymer. The polymers (polyurethanes) were obtained in a $30.8-71.2 \%$ yield

IR (Nujol): $v$ about $3300(\mathrm{~N}-\mathrm{H})$, about 1700 (amide I)

For partially soluble PU-b in chloroform, $\bar{M}_{n}$ is $7.4 \times 10^{\mathcal{3}}$ and $\bar{M}_{w} / \bar{M}_{n}$ is 1.59 .

\section{Characterization Techniques}

${ }^{1} \mathrm{H}$ NMR was carried out with a JEOL JPN-PMX60 spectrometer using $\mathrm{CDCl}_{3}$ as the solvent. DSC measurements were conducted with a Mettler 3000 series. WAXS patterns were recorded on a MAC Science $\mathrm{MXP}^{3} \mathrm{X}$-ray diffractometer equipped with a thermal controller model 5310. Optical microscopy was performed on a Nikon polarizing optical microscopy equipped with a Mettler FP80 controller and a FP82 hot stage. Gel permeation chromatography (GPC) was carried out with a Tosoh HLC-8020 instrument using chloroform as the eluent, equipped with four columns (TSK gel $\mathrm{G} 4000 \mathrm{H}_{\mathrm{HR}}$, $\mathrm{G} 3000 \mathrm{H}_{\mathrm{HR}}, \mathrm{G} 2000 \mathrm{H}_{\mathrm{HR}}$, and $\mathrm{G} 2000 \mathrm{H}_{\mathrm{HR}}$ ). The instrument was calibrated with a polystyrene standard.

Infrared spectroscopy data was recorded on a JEOL JIR-100 spectrometer. Spectra were collected at $4-\mathrm{cm}^{-1}$ resolution. A minimum of 20 scans was signal averaged. The measurement of thermally-controlled FT-IR spectra were performed as follows. Elevated temperature spectra of FT-IR were obtained by placing the cells made from two silicone wafers in a temperature-controlled cell. Silicone bases were prepared by washing the silicone wafers with an aqueous solution of hydrogen fluoride. Polyurethane thin films were placed between silicone wafers. The edges of the cells were sealed with an epoxy compound. The temperature was monitored via a thermocouple placed in the cell. Before each spectral acquisition the temperature was kept constant for $5 \mathrm{~min}$ to ensure complete temperature equilibration along the sample cell. At the end of the first heating run, the sample was cooled slowly to room temperature, followed by a second heating run in a similar fashion.

The Conditions of Curve-Fitting of the Amide I Band. The amide I band was resolved into three components according to these condition:

1. Three components were assigned to ordered hydrogen bonding $\mathrm{C}=\mathrm{O}$ peak, disordered hydrogen bonding $\mathrm{C}=\mathrm{O}$ peak, and free $\mathrm{C}=\mathrm{O}$ peak.
2. The base line was assumed to be from $1800 \mathrm{~cm}^{-1}$ to $1650 \mathrm{~cm}^{-1}$ (left to right).

3 . The range of curve-fitting agreed with that of the base line.

4. The shape of each peak was assumed to be Gaussian.

5. The curve-fitting procedure involved an error of $1 \%$.

\section{RESULTS AND DISCUSSION}

The SLCPUs were prepared by polyaddition of 2,2'iminodiethanol derivative (DM) with two kinds of diisocyanates. One was hexamethylene diisocyanate which was employed as a flexible alkyl chain in the polymer backbone (flexible SLCPU). The other one was $p$ phenylene diisocyanate which was used as a rigid core (rigid SLCPU). Scheme 1 shows the synthetic route for the rigid SLCPU. The flexible SLCPUs were not obtained by the same polymerization method. The diolmonomers in the previous works were prepared by the reduction of diethylmalonate derivatives. ${ }^{1-3}$ Consequently, we were not able to introduce many species of the mesogenic group in the SLCPUs. However, by the use of 2,2'-iminodiethanol instead of diethylmalonate derivatives, ${ }^{1-3}$ the rigid SLCPUs with various kinds of mesogenic group were easily synthesized. The rigid SLCPUs obtained were soluble in polar solvents such as tetrahydrofuran (THF) and dimethylformamide (DMF), but insoluble in chloroform except PU-b. Gel permeation chromatography (GPC) measurements for PU-b was carried out because the polymer was partially soluble in chloroform.

The thermal properties of 2,2'-iminodiethanol derivatives (DM) are shown in Table I. The 2,2'-iminodiethanol derivatives containing azobenzene (DM-a and b) and benzoate consisting of three benzene rings (DM-e) as a mesogenic core exhibited mesomorphic properties. In

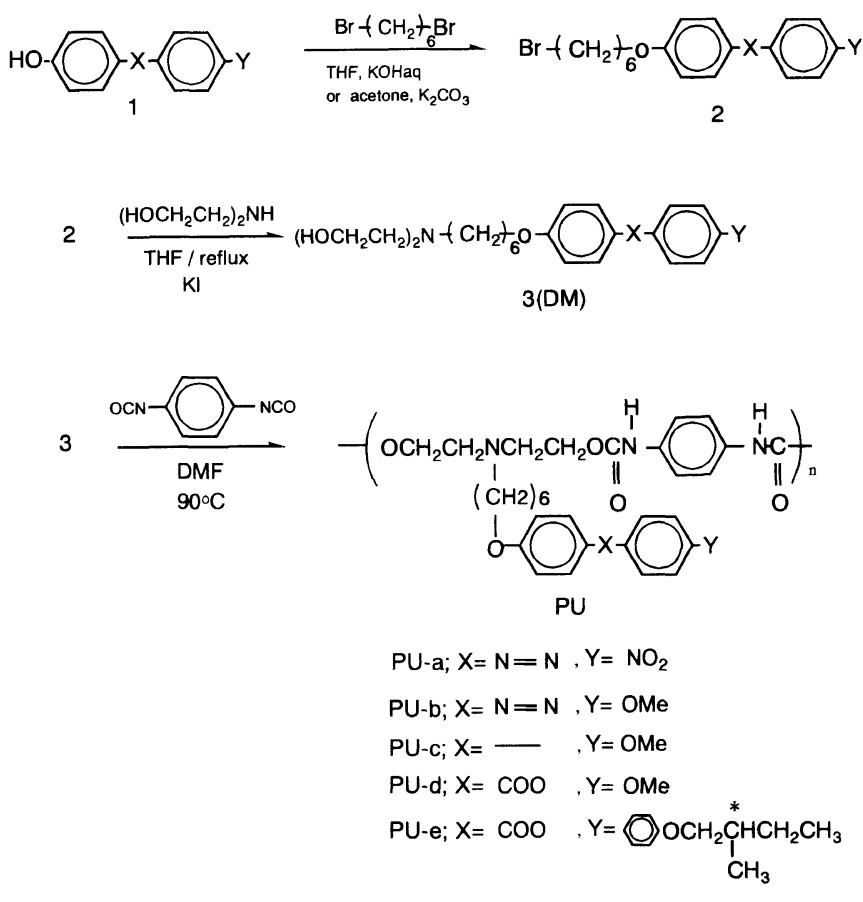

Scheme 1. Synthesis of diol-monomers and polyurethanes. 
Table I. Transition temperatures of diol-monomers

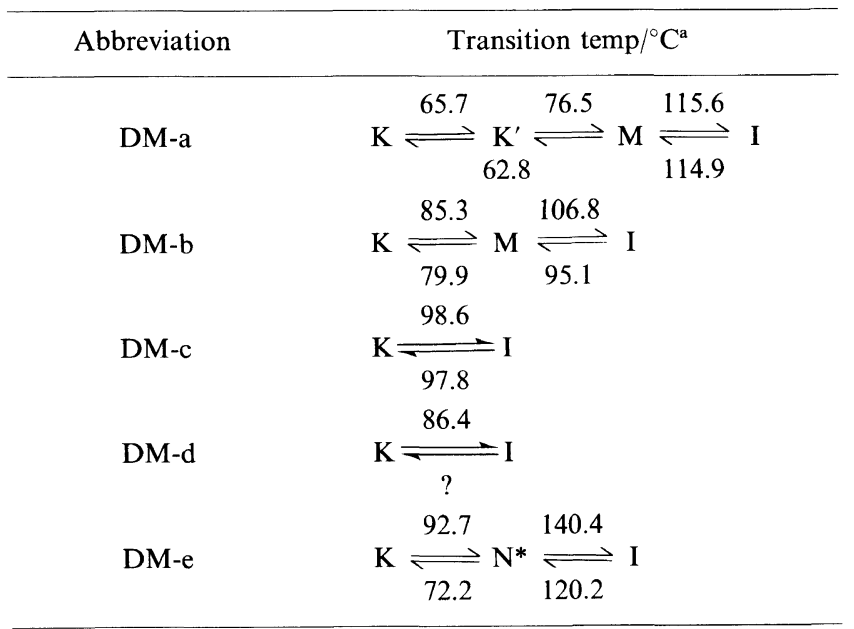

${ }^{a} \mathrm{~K}, \mathrm{~K}^{\prime}$, crystal; N, nematic; $\mathrm{M}$, mesophase; I, isotropic phase.

Table II. Transition temperatures of side chain type polyurethanes

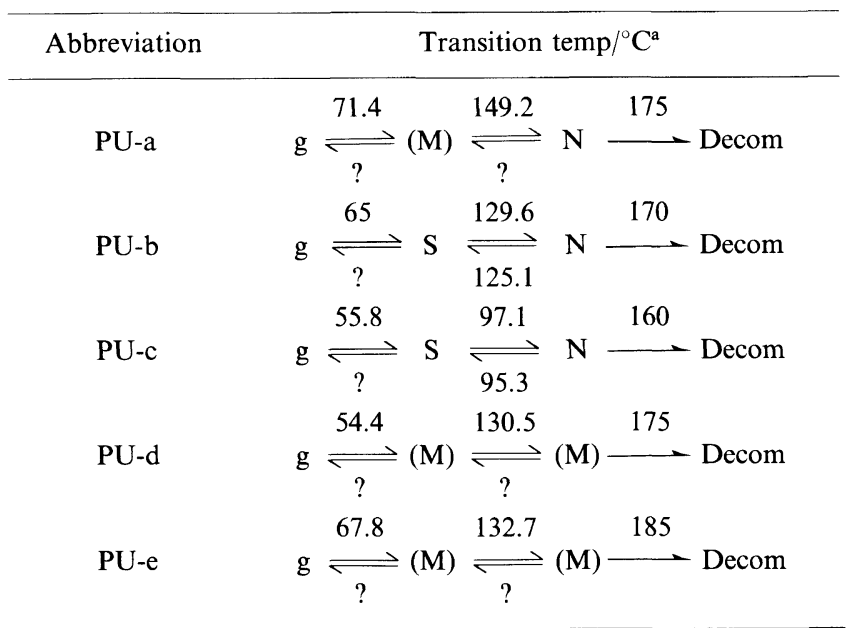

${ }^{a}$ g, glassy; S, smectic; N, nematic, M, mesophase.

particular, in the case of DM-e, an oily streak texture was observed by polarized light microscopy measurements. In the case of mesogenic groups with a methoxy group in the end group, the diol-monomer (DM-b) containing azobenzene core exhibited mesomorphic properties.

The thermal properties of the rigid SLCPUs are summarized in Table II. Three kinds of rigid SLCPUs (PU-a, b, and c) exhibited typical smectic and nematic phases. In the DSC curves of the rigid SLCPUs, two transition temperatures were observed. One was the glass transition temperature. The other one was attributed to the phase transition temperature. Transition enthalpies of these phase transitions for PU-a, b, and c were $1.6 \mathrm{~J} \mathrm{~g}^{-1}, 3.8 \mathrm{~J} \mathrm{~g}^{-1}$, and $1.7 \mathrm{~J} \mathrm{~g}^{-1}$, respectively. Typical $\mathrm{X}$-ray diffraction patterns for the rigid SLCPU are shown in Figure 2. We observed a diffraction peak in the small angle region $\left(2 \theta=2.06^{\circ}\right)$ corresponding to $42.8 \AA$ for the $d$-spacing, and a broad one in the wide angle region $\left(2 \theta=20.8^{\circ}\right)$ for PU-b below the transition temperature. The mesomorphic phase structure below the phase transition temperature was attributed to a smectic phase from the results of the X-ray diffraction pattern, DSC, and polarizing optical microscopy mea-

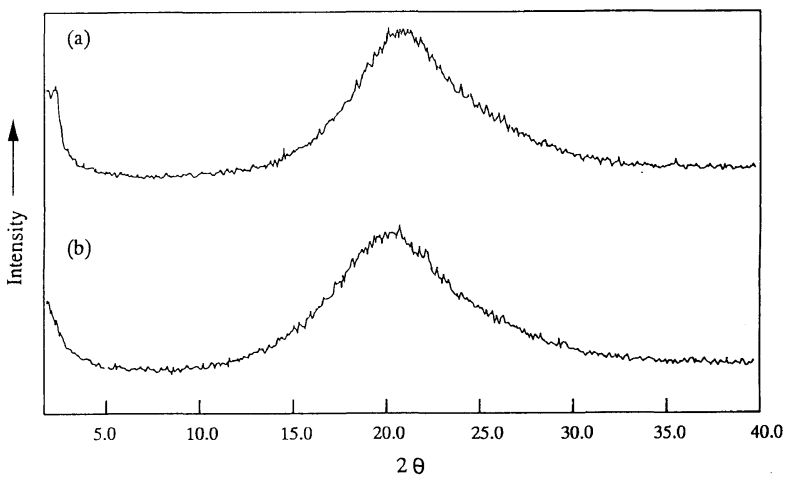

Figure 2. $\mathrm{X}$-Ray diffraction patterns of polymer (PU-b) at $77^{\circ} \mathrm{C}$ (a) and $146^{\circ} \mathrm{C}(\mathrm{b})$.

surements for PU-b. Only one broad diffraction peak was observed in wide angle region of the X-ray diffraction pattern above the phase transition for PU-b; therefore the mesomorphic phase structure was attributed to a nematic phase. We observed the same results of their measurements for PU-c. Birefringence for PU-a was observed above the glass transition temperature; however, in the X-ray pattern for PU-a between the glass transition temperature and the melting point, only one broad diffraction peak was observed in the wide angle region. The phase structure for PU-a for the temperature range was not clarified.

In DSC curves for the rigid SLCPUs containing benzoate type mesogenic groups (PU-d and e), we detected the glass transition temperature and the phase transition temperature. The peak attributed to the phase transition was very broad. Birefringence was observed above the glass transition temperature; however, the typical texture was not observed. Only one broad diffraction peak was detected in the wide angle region of the X-ray pattern above both the glass transition temperature and the melting point. We were not able to clarify the mesomorphic phase structures of these polyurethanes. Thermal decomposition occurred at 160$185^{\circ} \mathrm{C}$ for all polyurethanes.

Vibrational spectroscopy is one of the principal techniques for examining lateral interactions of the hydrogen bonding and conformational order in polymers. The specific lateral interaction occurring from $\mathrm{H}$-bonding was studied with thermally controlled FT-IR for main chain type liquid crystalline polyurethane, conventional polyamide and polyurethane. ${ }^{4-7}$ This technique was also applicable to the rigid SLCPUs.

On the heating run, new peaks for PU-a, $b$, and c were observed at the higher wavenumber of $3300 \mathrm{~cm}^{-1}$ with increasing temperature. These peaks were attributed to free $\mathrm{NH}$ stretching vibration. These results indicated thermal dependence in the strength of H-bonding. On the other hand, the amide I band for PU-b and c shifted to higher wavenumbers with increasing temperature as shown in Figure 3. The shift tendency was dependent on the species of the mesogenic group. The gradual increase in wavenumber of the amide I band for PU-b was observed with increasing temperature to the phase transition temperature. A large shift of the amide I band for PU-b was shown near the phase transition temperature. The results indicated a gradual decrease in the strength of $\mathrm{H}$-bonding with increasing temperature 


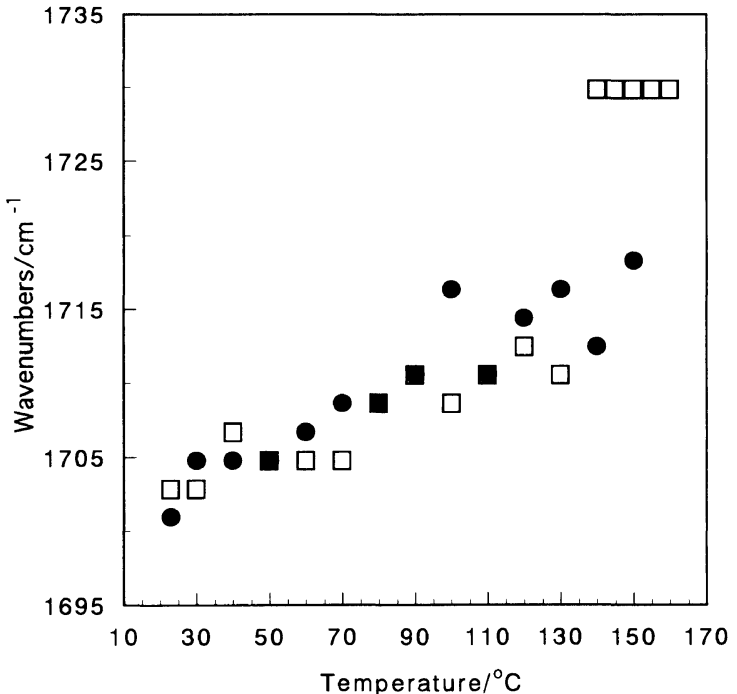

Figure 3. Variation of the band top of the amide I region of polymers [PU-b ( $\square)$ and PU-c (๑)].

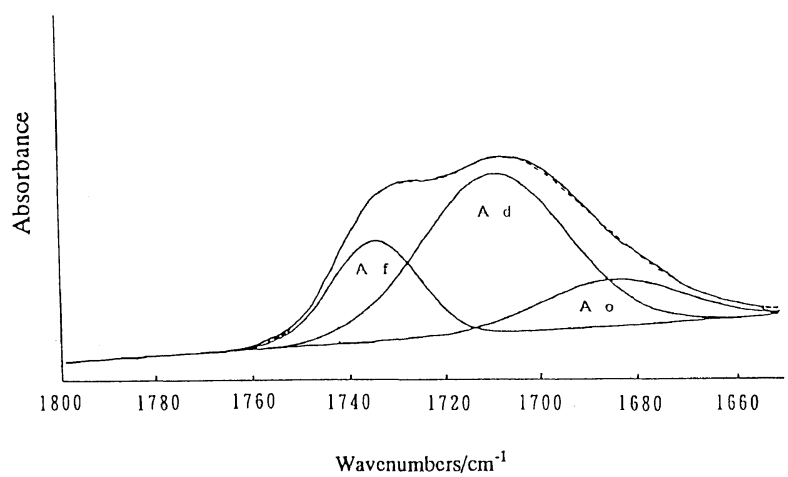

Figure 4. Least squares deconvolution of the amide I band for PU-c.

and a remarkable decrease in it at the phase transition temperature. A nematic phase was shown with remarkable decrease in the strength of $\mathrm{H}$-bonding. The amide I band for PU-c shifted gradually to higher wavenumbers with increasing temperature. No remarkable shift was observed at the phase transition temperature compared with PU-b. These results demonstrated that a nematic phase for PU-c was exhibited with a small decrease in the strength of $\mathrm{H}$-bonding.

Further in order to clarify the thermal dependence of $\mathrm{H}$-bonding for the rigid SLCPUs, each spectrum of the amide I band recorded at different temperatures was divided into three components such as ordered hydrogen bonded carbonyl groups $\left(\mathrm{A}_{\mathrm{o}}\right)$, disordered hydrogen bonded carbonyl groups $\left(\mathrm{A}_{\mathrm{d}}\right)$, and non-hydrogen bonded (free) carbonyl groups $\left(\mathrm{A}_{\mathrm{f}}\right)$, as shown in Figure 4.

The thermal dependence of the peak area for each component is shown in Figure 5. $A_{o}$ decreased gradually on the heating run, while $A_{d}$ and $A_{f}$ increased with increasing temperature. The change in these peak areas showed a gradual decrease in the strength of $\mathrm{H}$-bonding with increasing temperature. Mesomorphic properties were exhibited with a gradual decrease in the strength of H-bonding. The same result was observed for PU-c; however, the magnitude of the change in the strength of H-bonding was smaller than that of PU-b. The magnitude was dependent on the species of the mesogenic group. This result was the same as that of the shift

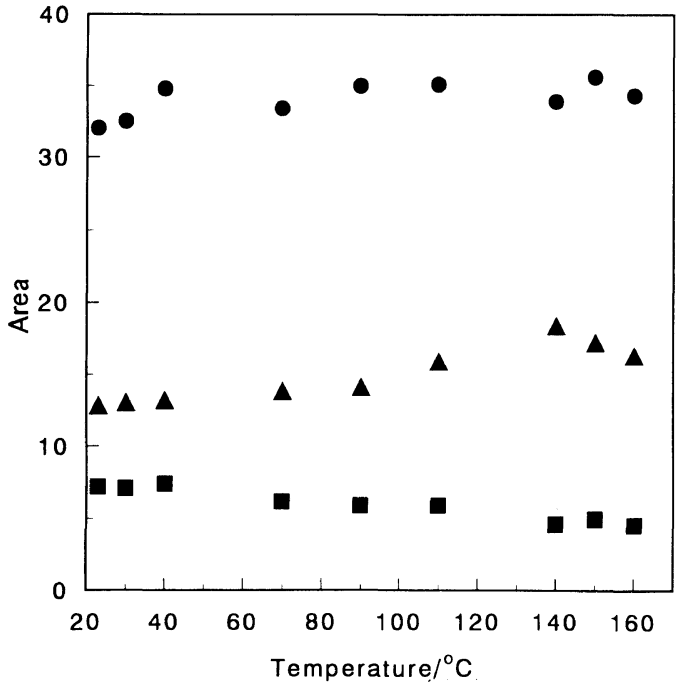

Figure 5. Plots of the area of the free $\left(A_{f}, \mathbf{\Delta}\right)$-, disordered $\left(A_{d}, \mathbf{O}\right)$-, and ordered $\left(A_{0}, \square\right)$ - hydrogen bonding $C=O$ peaks as a function of temperature.

tendency of the amide I band.

We have already reported that the strength of $\mathrm{H}$ bonding for rigid SLCPUs does not change drastically near the phase transition temperature. ${ }^{1-3}$ But we observed a thermal dependence in the strength of $\mathrm{H}$ bonding for these rigid SLCPUs. The difference of the change in the strength of $\mathrm{H}$-bonding resulted from the difference of flexible chain length in the polymer backbone.

\section{CONCLUSIONS}

Three kinds of rigid SLCPUs exhibited typical smectic and nematic phases. The thermal dependence in the strength of $\mathrm{H}$-bonding was observed for the rigid SLCPUs. Mesomorphic properties were exhibited with a gradual decrease in the strength of $\mathrm{H}$-bonding with increasing temperature.

\section{REFERENCES}

1. M. Portugal, H. Ringsdorf, and R. Zentel, Makromol. Chem., 183, 2311 (1982).

2. B. Hahn, H. Wendorf, M. Portugal, and H. Ringsdorf, Colloid. Polym. Sci., 59, 875 (1981).

3. H. Finkelmann and G. Rehage, Makromol. Chem., Rapid Commun., 1, 733 (1980).

4. M. Tanaka and T. Nakaya, Adv. Urethane Sci. Technol., 12, 1 (1993).

5. P. Pieter, E. W. Petrus, and J. Bernard, Eur. Pat. Appl. EP550105 (21 December 1992).

6. E. Akiyama and N. Koide, Liquid Crystals, 14, 1645 (1993).

7. T. Mihara, E. Akiyama, and N. Koide, Rep. Prog. Polym. Phys. Jpn., 36, 259 (1993).

8. N. Koide, E. Akiyama, and T. Mihara, Mol. Cryst. Liq. Cryst., 254, 283 (1994).

9. D. J. Skrovanek, S. E. Howe, P. C. Painter, and M. M. Coleman, Macromolecules, 18, 1676 (1985).

10. D. J. Skrovanek, P. C. Painter, and M. M. Coleman, Macromolecules, 19, 699 (1986).

11. M. M. Coleman, K. H. Lee, D. J. Skrovanek, and P. C. Painter, Macromolecules, 19, 2149 (1986).

12. F. Papadimitrakopoulos, E. Sawa, and W. J. MacKnight, Macromolecules, 25, 4682 (1992). 